\title{
CPR I 30 Frgm. i wiederentdeckt
}

$\mathrm{B}^{\prime}$

EI der angeblichen "Invocatio ", die in CPR 1 30.i steht, handelt es sich um eine im Vergleich zu den sonst bekannten Invokationsformeln, die die Heilige Trinität oder Jesus Christus anrufen und die wir aus griechischen und koptischen dokumentarischen Papyri kennen, völlig abweichende Formel ( ${ }^{(1)}$.

Nach Ansicht der Autoren kb'nnte der besagte Text am Beginn von Frgm. i statt mit einer Invocatio mit einer Art Gebet vel similiter beginnen, wie beispielsweise P. Ness. III 89.45, P. Oxy. VIII $1151.40 \mathrm{ff}$. oder SB III 6087. $15 \mathrm{ff} .\left({ }^{2}\right)$. zeigen. Jedenfalls war dieser Papyrus jedoch bis auf ein kleines Fragment aus der Glasplatte verschwunden, in der er zu C. Wesselys Zeiten aufbewahrtworden war, und dieses Verschwinden machte es auch unmöglich, die Lesungen der editio prineeps (cf. M. Chrest. 290) und deren Korrekturen in BL I 117 zu überprüfen.

Dr. H. Harrauer hat nun den « verlorenen " Teil von $C P R$ I 30 Frgm. i als P. Vindob. G 37 identifizieren können. Ihm verdanken wir es, dass wir in einigen Punkten über die editio princeps hinausgehen kbnnen :

a. Wesselys Text ist im wesentlichen korrekt. Einige Verbesserungen

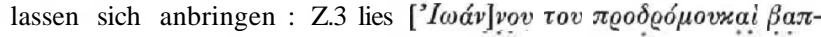

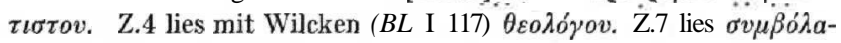

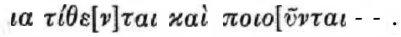

b. In Z.5 gibt es zwischen $\mu \alpha \varrho \tau v \varrho \omega \nu$ und dem folgenden $\tau \alpha \pi \alpha \varrho o v \tau \alpha$ einen kleinen Zwischenraum, aber es gibt tatsächlich keine Spur einer Datierungsformel auf dem Papyrus, wie man sie sonst auf einem normalen rechtsverbindlichen Dokument findet.

c. Der Text auf CPR I 30 Frgm. $\mathbf{i}^{\mathbf{R}}$ ist gegen die Fasern geschrieben, wie auch der andere, grbssere als CPR I 30 Frgm. ii veröffentlichte Text, der eigentliche Heiratsvertrag (P. Vindob. G 19995); beide sind transversa charta $\left({ }^{3}\right)$ geschrieben. Beide Teilen haben aber

(1) S. dazu R. S. Bagnall und K. A. Worp, Christian Invocations in the Papyri, Cd'E 56 (1981), 112ff., bes. 131-133; 362-365.

(2) Vgl. in dieser Zusammenhang auch das christliche Gebet in P. Bad. IV 65.

(3) Vgl. E. G. Turnen, The Terms Recto and Verso. The Anatomy of the Papyrus Roll (Brüssel 1978), 26ff. 
nichts mit einander zu tun, weil sie von verschiedenen Schreibern geschrieben sind.

Aber auch die Wiederauffindung des Papyrus bringt uns einer Entscheidung pro oder contra, ob wir es hier mit einer abweichenden Invocatio oder mit einer Art Gebet zu tun haben, nicht näher.

Zur Losung dieser offenen Frage tragt auch nicht die Auffindung eines weiteren ahnlichen Fragments in der Wiener Papyrussammlung durch J. M. Diethart bei, das im folgenden mitgeteilt wird :

P. Vindob. G 25816

$=S B 16$,

15 x $18,7 \mathrm{~cm}$.

Herkunft unbekannt

vi. oder viI. Jh.

Mittelbrauner Papyrus, oben, rechts und unten abgebrochen, links ein Freirand zwischen 5,9 und 7,4 cm. Die Schrift geht parallel zu den Fasern. Braune Tinte. Verso leer.

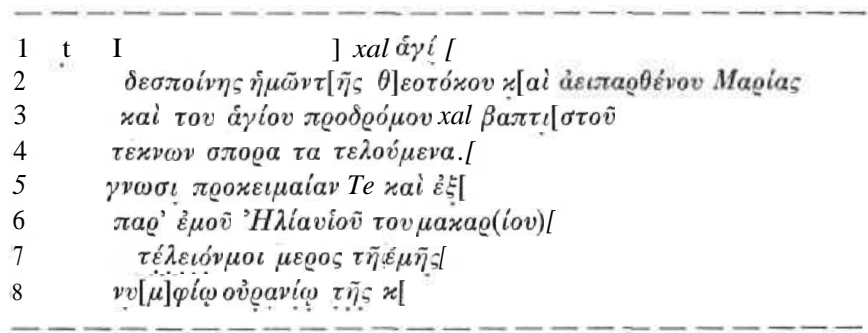

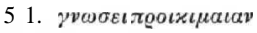

$G$ Pap. vlov

Auch bei diesem Fragment haben wir es offensichtlich mit einem Dokument, das Eheangelegenheiten betrifft, zu tun : vgl. Z.4, $\tau \varepsilon \varkappa v \omega \nu \sigma \pi о \varrho a$

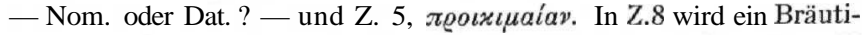

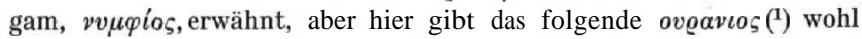
an, dass hier Jesus Christus gemeint ist $\left.\mathbf{(}^{2}\right)$. Eine befriedigende Erklärung fiir diese Anspielung auf Jesus Christus als "himmlischer Bräutigam » können wir nicht geben, und überhaupt ist uns eine exakte Deu-

(1) Aus der Wortstellung erhellt u.E., dass kaum mit dem Personennamen Oboúvıos zu rechnen ist.

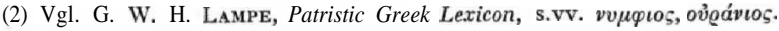


tung des Fragments nicht gelungen. Ausser um einen Ehekontrakt kann es sich auch z.B. um ein Dokument handeln, in dem es beispielsweise um eine Scheidung oder um eine Erbschaftsangelegenheit geht. War der "Himmlische Bräutigam" (Z.8) mit einer Nonne, die Partei in einer Erbschaftsangelegenheit war, verheiratet?

Es ist jedenfalls auffällig und interessant genug, dass dieses Fragment in den ersten zwei Zeilen ähnliche Formulierungen enthält, wie sie in CPR I $30 \mathrm{Frgm}$. i stehen. Vorausgesetzt, dass Z.2 nicht länger war als unsere gegenwärtige Erganzung, musste allerdings die vorliegende Formel kürzer sein als die im $C P R$ I-Text $\left({ }^{1}\right)$.

Wien

Amsterdam

\section{J. M. DIETHART}

K. A. WorP

(i) Unser besonderer Dank gilt Frau Hofrat Dr. Helene Loebenstein, Direktor der Wiener Papyrussammlung, fur die entgegenkommende Erteilung der Publikationserlaubnis. Dr. H. Harrauer danken wir fur wertvolle Hinweise und Anregungen. 